\title{
Social Policy Digest Online
}

The Social Policy Digest is a major online resource providing up-to-date listings of developments across the whole range of UK and pan-European social policy issues - including links to source materials and commentary. The Digest is published by Cambridge University Press on behalf of the Social Policy Association.

The Digest is a freely accessible resource. Simply go to: http://journals. cambridge.org/spd/action/home. The database of material dates back to 2002 . By the end of 2014, it contained over 43,00o separate items.

New Digest items are posted on a near-daily basis to the continuous 'Latest News' listing on the Digest homepage.

To be notified of Digest updates automatically, simply use one of the news feeds provided (RSS or Atom). Digest updates are also available via Facebook and Twitter.

Subject categories in the Digest database include: poverty, inequality and social security; unemployment and work; education; health; social services and care; housing; local communities and services; crime and justice; and voluntary activity and civil society. Cross-cutting categories include: children and the family; disability; gender and sexuality; mental health; old age; race and ethnicity; and young people. There are also listings relating to social policy developments in Scotland, Wales and Northern Ireland. Each entry provides a summary of the source document, together with source references and online links.

We hope that you will visit the online Digest, and that you will find it useful. We welcome feedback via the email address given on the Digest site.

\section{Jacqui Croft}

